\title{
AVALIAÇÃo DOS TRATAMENTOS DE SUPERFÍCIE NA RESISTÊNCIA DE UNIÃO ENTRE DOIS PINOS DE FIBRA DE VIDRO E UMA RESINA COMPOSTA
}

\author{
Evaluation of surface treatments on the bond strength \\ between two fiberglass posts and a composite resin
}

Andreza Barbosa Santos Guimarães ${ }^{a}$, (DRana de Brito Granjaa , (DEmily Vivianne Freitas da Silva ${ }^{b}$, (D) Roberta Novisc, (D)Blanca Liliana Torres Léon ${ }^{d}$

\section{RESUMO}

Introdução: O objetivo deste trabalho foi avaliar a influência de diferentes métodos de tratamento de superfície em pinos de fibra de vidro com relação a sua força de união com a resina composta e avaliar os tipos de falhas ocorridas. Materiais e Métodos: Foram utilizados 36 pinos da WhitepostDC (grupo GF) e Exacto Cônicos (grupo GA), divididos em: Grupos controle GF-1 e GA-1 - aplicação de silano de acordo com as especificações do fabricante; Grupos GF-2 e GA-2 - condicionamento com peróxido de hidrogênio a 10\% e silanização; Grupos GF-3 e GA-3 - Jateamento com óxido de alumínio por 10 segundos e silanização. A adição da resina composta foi realizada em incrementos de $2 \mathrm{~mm}$, com fotopolimerização de cada incremento por 40 segundos, até o preenchimento de toda extensão da matriz. Então, o conjunto pino e resina composta foi seccionado em três porções (cervical, médio e apical) $(\mathrm{n}=18)$. As amostras foram submetidas à análise de resistência de união através do teste Push-Out em uma Máquina de Ensaio Universal (EMIC), e os tipos de falhas foram avaliados com aumento $30 \times$ com um microscópio óptico. Os dados foram submetidos à análise de variância, ao teste de Tukey e ao teste de qui-quadrado com significância de 5\%. Resultados: Nos grupos GA, o tratamento com silano (23,10 $\mathrm{MPa})$ resultou em resistência de união estatisticamente maior que os tratamentos com peróxido (17,19 $\mathrm{MPa})$ e com jateamento (16,28 $\mathrm{MPa})$. No tratamento com peróxido, a resistência de união foi estatisticamente maior no terço médio $(21,16 \mathrm{MPa})$ que no terço apical (12,55 MPa). A falha do tipo mista teve a maior prevalência (57,01\%). Conclusão: O tratamento com silano apresentou maior resistência de união, com diferença estatística dos demais grupos na marca Angelus.

Palavras-chave: Pinos dentários. Resinas compostas. Propriedades de superfície.

\section{ABSTRACT}

Introduction: The aim of this study was to evaluate the influence of different surface treatment methods on glass-fiber posts relative to their bond strength with the composite resin and to evaluate the types of failures that occurred. Materials and Methods: Thirty-six posts from WhitepostDC (FG group) and Exacto Conics (AG group) were used, divided into: Control FG-1 and AG-1 groups - application of silane, according to manufacturers' specifications; FG-2 and AG-2 groups - conditioning with $10 \%$ hydrogen peroxide and silanization; FG-3 and AG-3 groups - aluminum oxide blasting for 10 seconds and silanization. The composite resin was added in $2-\mathrm{mm}$ increments, each increment was photopolymerized for 40 seconds, until the entire length of the matrix was filled. Then, the assembly (fiber post and composite resin) was sectioned into three portions (cervical, middle and apical) $(\mathrm{n}=18)$. Samples were submitted to the bond strength analysis by a push-out test in a Universal Testing Machine and the failure types were evaluated with a $30 \times$ magnification with an optical microscope. Data were

\footnotetext{
${ }^{\text {a} G r a d u a d a ~ e m ~ O d o n t o l o g i a ~ p e l a ~ U n i v e r s i d a d e ~ F e d e r a l ~ d a ~ B a h i a ~-~ U F B A, ~ S a l v a d o r, ~ B A, ~ B r a s i l . ~}$

' Pós-doutoranda em Prótese Dentária pela Faculdade de Odontologia de Araçatuba - Universidade Estadual Paulista, Araçatuba, SP, Brasil. 'Doutorando em Prótese Dentária pela Universidade São Leopoldo de Mandic, Campinas, SP, Brasil.

${ }^{d}$ Professora Associada da Universidade Federal da Bahia - UFBA; professora Adjunta da Escola Bahiana de Medicina e Saúde Pública - EBMSP; professora do Curso de Pós-Graduação - EBMSP, Salvador, BA, Brasil.
}

Autora de correspondência: Dra. Blanca Liliana Torres León - E-mail: blalitole@hotmail.com

Data de envio: $31 / 10 / 2020$ | Data de aceite: 30/12/2020 
submitted to the analysis of variance, the Tukey test, and the chi-square test with a 5\% significance level. Results: For AG groups, the treatment with silane (23.10 $\mathrm{MPa}$ ) resulted in statistically greater bond strength than the treatments with peroxide (17.19 $\mathrm{MPa}$ ) and blasting (16.28 $\mathrm{MPa})$. It was found that the bond strength was statistically higher in the middle third (21.16 MPa) than in the apical third (12.55 MPa) for the peroxide treatment. The mixed-type failure had the highest prevalence (57.01\%). Conclusion: Silane treatment showed statistically greater bond strength values, when the Angelus brand was tested.

Keywords: Dental pins. Composite resins. Surface properties.

\section{INTRODUÇÃO}

Os pinos de fibra de vidro são uma alternativa ao núcleo metálico fundido, com adequadas propriedades biomecânicas, como módulo de elasticidade semelhante ao da dentina. Além disso, são pinos estéticos de fácil técnica operatória e fácil remoção, caso necessário'. A longevidade das reabilitações com pinos de fibra de vidro depende não apenas das características mecânicas, como dureza e resistência a flexão, mas também da sua adesão com o material resinoso e estrutura dentária ${ }^{1,2}$.

Na revisão sistemática elaborada por Marchionatti et al. ${ }^{3}$, foram analisados 11 artigos clínicos sobre uso de pinos para reabilitação. Os pinos de fibra apresentam boa durabilidade e comportamento clínico, com taxa de sucesso de 71 a 100\% em um tempo de acompanhamento de 19,2 a 109 meses. Já Figueiredo et al. ${ }^{4}$, em uma revisão sistemática de 14 estudos observaram uma taxa de sucesso de $83,9 \%$ dos pinos de fibra acompanhados por um tempo mínimo de 5 anos. Embora esse método restaurador apresente boa durabilidade clínica, falhas podem ocorrer, sendo a adesão do pino no interior do canal radicular o ponto mais crítico da restauração. Assim, o deslocamento do pino no interior do canal radicular é a falha mais comum, podendo contribuir para a ocorrência de fratura radicular. Outra falha comum é a falha da resina composta no núcleo de preenchimento. Desta forma, o sucesso da restauração está na relação de união entre os compósitos resinosos, substrato dentinário e pino de fibra de vidro ${ }^{5,6}$.

Para otimizar a resistência de união do núcleo de preenchimento de resina composta aos pinos de fibra, vários tratamentos de superfície têm sido propostos para proporcionar maior adesão química e micromecânica ${ }^{6}$. O tratamento de superfície mais comumente utilizado é a aplicação de silano no pino de fibra, porém é um tratamento de resistência relativamente baixa, quando comparado ao uso do peróxido de hidrogênio a $10 \%{ }^{7}$. Com o tratamento com silano, há ausência da união entre o mesmo e a matriz de resina epóxica que recobre as fibras dos pinos $^{7-9}$.

Dessa forma, tratamentos de superfícies são propostos na literatura para retirar a camada de resina epóxica superficial das fibras dos pinos e, consequentemente, aumentar a sua união à resina composta ${ }^{1,2}$. Como exemplo, há o jateamento com partículas de óxido de alumínio ou sílica e condicionamento com ácido fluorídrico. Porém, não são procedimentos totalmente confiáveis, pois podem ocasionalmente gerar danos às fibras de vidro do pino ${ }^{9-11}$. Então, outros mecanismos químicos foram propostos, como o uso de permanganato de potássio, peróxido de hidrogênio e etóxido de sódio ${ }^{1}$. Entre essas substâncias, o tratamento de superfície com peróxido de hidrogênio é o que tem apresentado melhores resultados devido à sua fácil técnica operatória ${ }^{1}$ e dissolução parcial da resina epóxica, sem danos às fibras do pino ${ }^{10}$.

Essa dissolução parcial expõe a superfície de fibra para silanização $0^{1,2,7}$. Porém, ainda não há um protocolo definido ou uma padronização do condicionamento do pino de fibra com peróxido de hidrogênio em relação ao tempo de utilização, concentrações adequadas, ação 
produzida por esse tratamento e a necessidade de substâncias química adicional ${ }^{6}$. Portanto, o objetivo deste trabalho foi avaliar a resistência de união entre resina composta e dois tipos de pinos de fibra de vidro após a aplicação de diferentes tratamentos de superfície e analisar os tipos de falhas ocorridas nos corpos-de-prova após o teste push-out. A hipótese nula do estudo foi que os diferentes tratamentos de superfície avaliados não interferem na resistência de união entre resina composta e os pinos de fibra de vidro.

\section{MATERIAIS E MÉTODOS}

Foram utilizados 36 pinos de fibra de vidro, divididos em WhitepostDC (FGM, Joinville, SC, Brasil) (grupo GF, comprimento de $20 \mathrm{~mm}$ ) e Exacto Cônicos (Angelus, Londrina, PR, Brasil) (grupo GA, comprimento de $17 \mathrm{~mm}$ ), íntegros, com dupla conicidade e superfície lisa. Os pinos foram divididos em três grupos cada, sendo: GF-1 (controle): Silano PROSIL; GF-2: Peróxido de hidrogênio a 10\% + silano; GF-3: Jateamento com óxido de aluminio + silano; GA-1 (controle): Aplicação de silano + aplicação de adesivo fusion duralink catalisador; GA-2: Peróxido de hidrogênio a 10\% + silano; GA-3: Jateamento com óxido de alumínio + silano (Fluxograma 1).

Fluxograma 1: Distribuição das amostras do estudo.

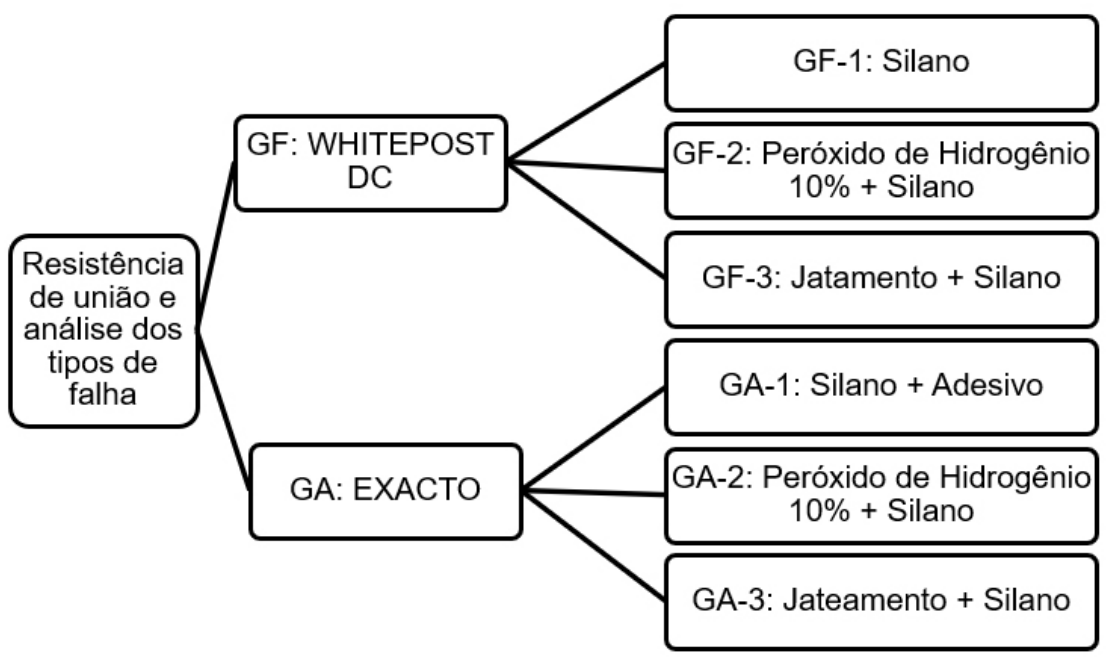

Para o tratamento de superfície, todos os pinos foram imersos em álcool 70\% por 60 segundos. Nos grupos GF-1 e GA-1, os pinos foram submetidos aos procedimentos indicados pelos fabricantes. Em GF-1, foi aplicado o silano (Prosil - FGM, Joinville, SC, Brasil) e aguardou-se a secagem por 60 a 120 segundos. Em GA-1, foi realizada a aplicação de silano, deixando-o secar por 60 segundos, seguido da aplicação do adesivo Fusion-Duralink Catalisador (Angelus, Londrina, PR, Brasil) com auxílio de microbrush e fotoativação (Ultralux El, Dabi Atlante, Ribeirão Preto, SP, Brasil) por 10 segundos na intensidade de $500 \mathrm{~mW} / \mathrm{cm}^{2}$ Para os grupos GF-2 e GA-2, os pinos foram imersos em peróxido de hidrogênio a 10\% (Alfaparf Milano, Milão, BG, Itália) por 10 minutos em temperatura ambiente. Então, os pinos foram lavados com água destilada (30 mL) com o auxílio de uma seringa e secados com jatos de ar durante 60 segundos. Em seguida, o silano foi aplicado, deixando-o secar por 60 segundos. Nos grupos GF-3 e GA-3, foi realizado o jateamento com micropartículas de óxido de alumínio durante 10 segundos à uma distância de $10 \mathrm{~mm}$ e com pressão de 100 libras, utilizando uma máquina de jateamento (Precision 1- Essence Dental, Araquara, SP, Brasil) em laboratório. 
Os pinos foram girados manualmente para garantir que toda superfície seria jateada. Em seguida os pinos passaram pelo processo de silanização e secagem dos mesmos por 60 segundos.

Para confecção dos corpos-de-prova, foi utilizada uma matriz metálica bipartida contendo um orifício cilíndrico central principal de $10 \mathrm{~mm}$ de altura e $5 \mathrm{~mm}$ de diâmetro e um orifício secundário central a este, também cilíndrico, de $2 \mathrm{~mm}$ de altura e diâmetro (Figura 2). Os pinos foram adaptados ao centro da matriz metálica com o auxílio de uma pinça, sendo utilizada cera utilidade (Lysanda, São Paulo, SP, Brasil) para garantir maior estabilização.

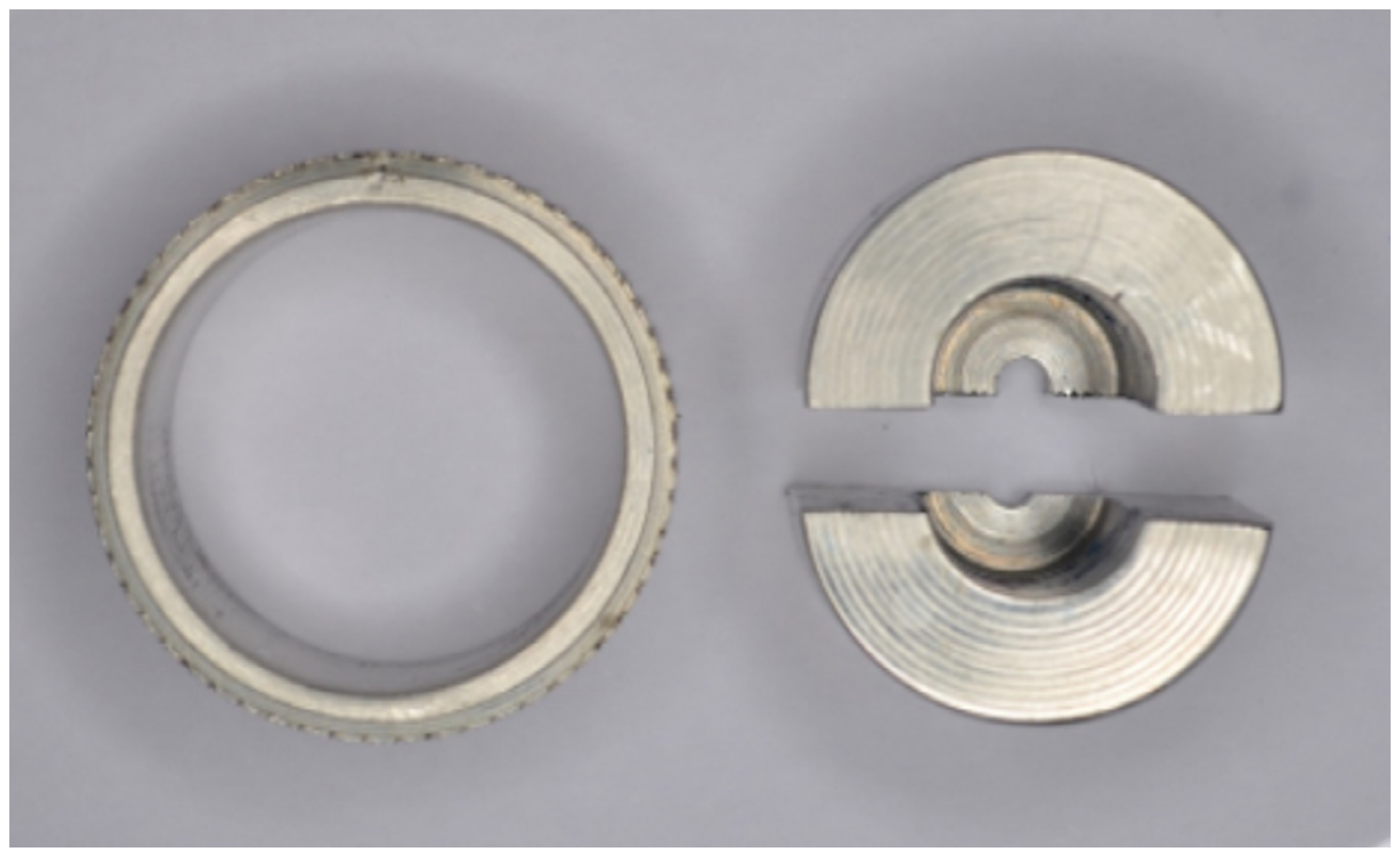

Figura 1: Matriz metálica bipartida.

Lateralmente aos pinos, preenchendo a matriz por completo, foram inseridas camadas de resina Opallis cor DA2 (FGM, Joinville, SC, Brasil), com incrementos de $2 \mathrm{~mm}$ de altura (seguindo marcações contidas no interior da matriz), com o auxílio de uma espátula de inserção e de um calcador para uma melhor acomodação da resina no protótipo metálico. Cada camada foi fotopolimerizada durante 40 segundos, até o preenchimento de toda a extensão da matriz (Figura 2). Então, os corpos-de-prova foram removidos e seccionados transversalmente (3 mm de altura) com discos diamantados bilaminados (Fava, São Paulo, SP, Brasil) (Figura 3). Cada secção resultou em três amostras correspondentes aos terços apical (A), médio (M) e cervical (C), totalizando 108 amostras. Foi realizado o acabamento dos espécimes com discos de lixa (TDV, Pomerode, SC, Brasil). Previamente à execução do ensaio, a metodologia foi testada e ajustada, através da realização de um ensaio piloto. 


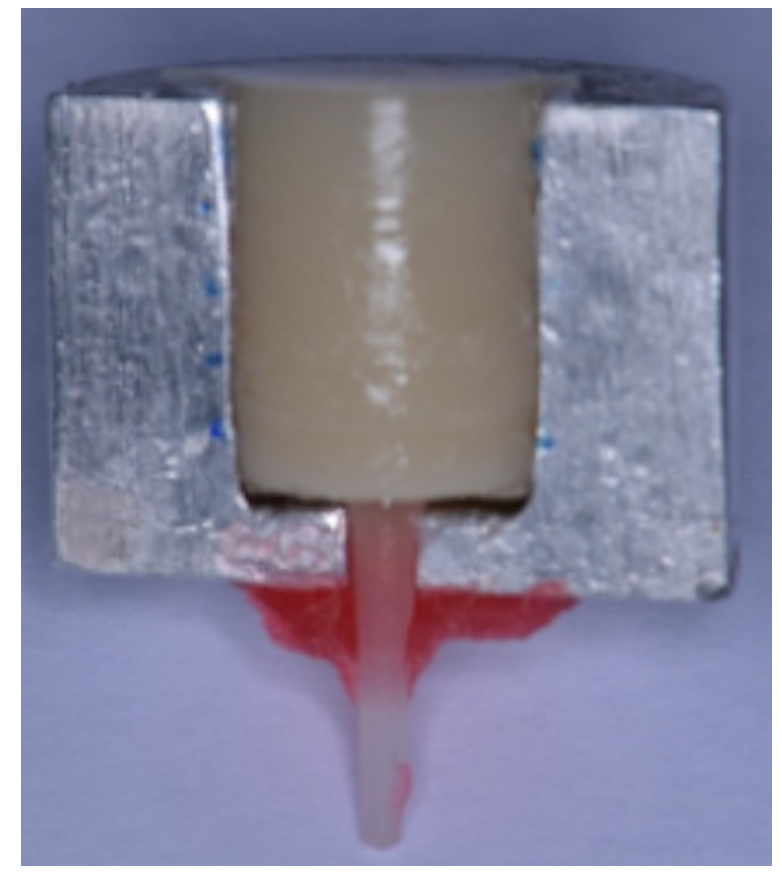

Figura 2: Confecção do corpo de prova.

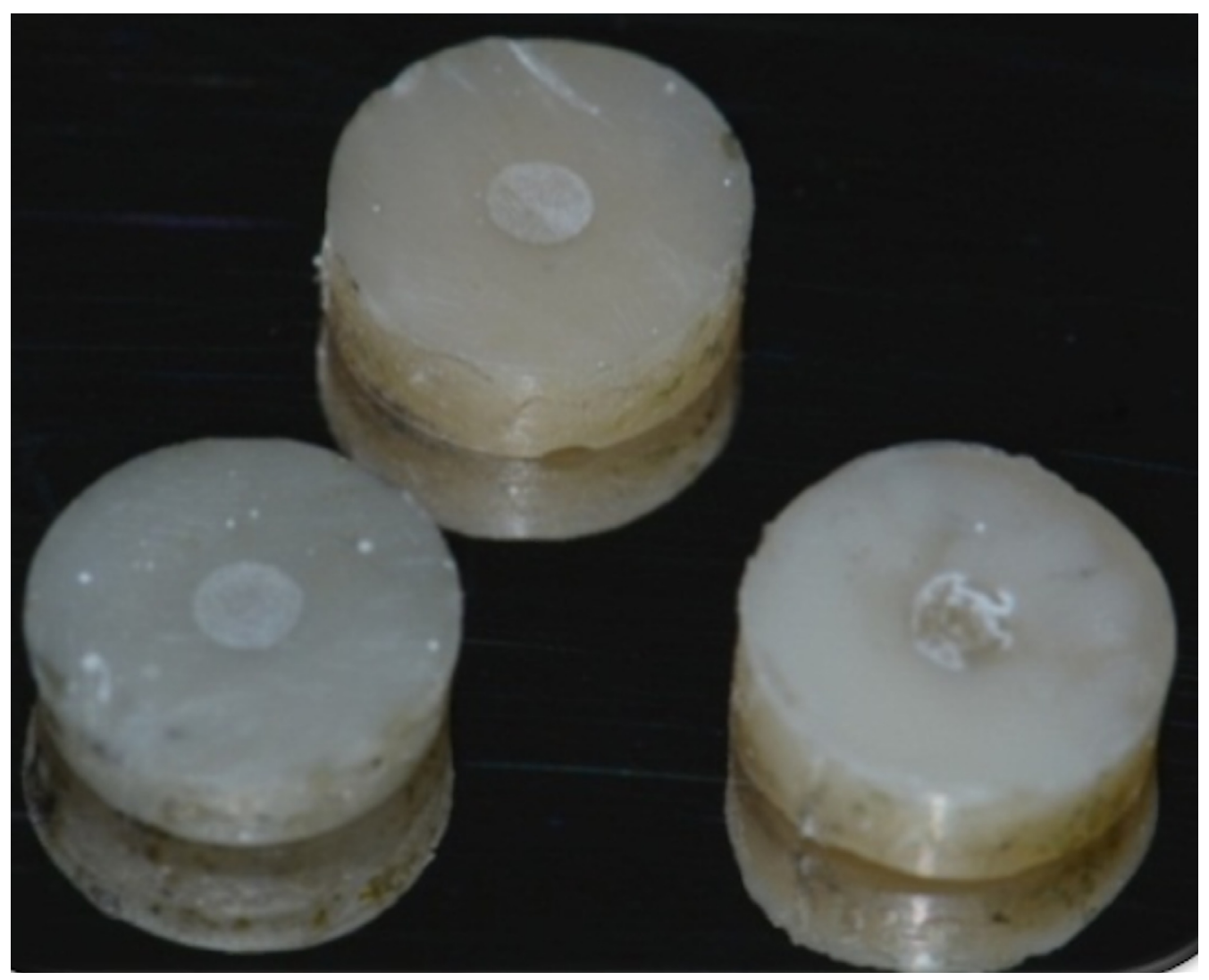

Figura 3: Corpo de prova seccionado. 
Previamente ao início do ensaio, a amostra foi mantida em água destilada a $37^{\circ} \mathrm{C}$ durante 24 horas. Para a realização da análise da resistência de união, a amostra de cada grupo ( $\mathrm{n}=18)$ foi submetida ao teste Push-Out em uma Máquina de Ensaio Universal (EMIC, São José dos Pinhais, PR, Brasil). Uma haste metálica com ponta ativa de 1,0 $\mathrm{mm}$ de diâmetro foi fixada no mordente da máquina (EMIC DL $500 \mathrm{MF}$ ) com uma célula de carga de $50 \mathrm{Kg}$ e posicionada no centro do pino de fibra de vidro. O teste de push-out foi realizado a uma velocidade média de 0,5 mm/min até o deslocamento da peça. Para determinar a resistência de união em Mega Pascal ( $\mathrm{MPa}$ ), os valores da força máxima em Newton (N) foram divididos pela área da interface de união. O cálculo foi feito por meio da seguinte fórmula: $\mathrm{MPa}=\mathrm{N} / 2 \pi \mathrm{rh}$, onde $\mathrm{r}$ é o raio do pino, h a altura da amostra em milímetros e $\pi$ equivale a $3,14^{11}$. A análise do tipo de falha foi realizada com o uso de um microscópio óptico com aumento $30 \times$ (Maquira, Maringá, PR, Brasil), sendo as falhas distribuídas em: coesivas do pino (falha localizada dentro da estrutura do pino), coesivas da resina (falha localizada dentro da estrutura da resina composta), adesiva (falhas na interface entre pino e resina composta) ou mistas (quando mais de um tipo de falha pode ser visualizada em uma mesma amostra) ${ }^{1}$ (Figura 4). Os resultados foram submetidos à análise de variância e as médias foram comparadas utilizando o teste de Tukey com 5 \% de significância.
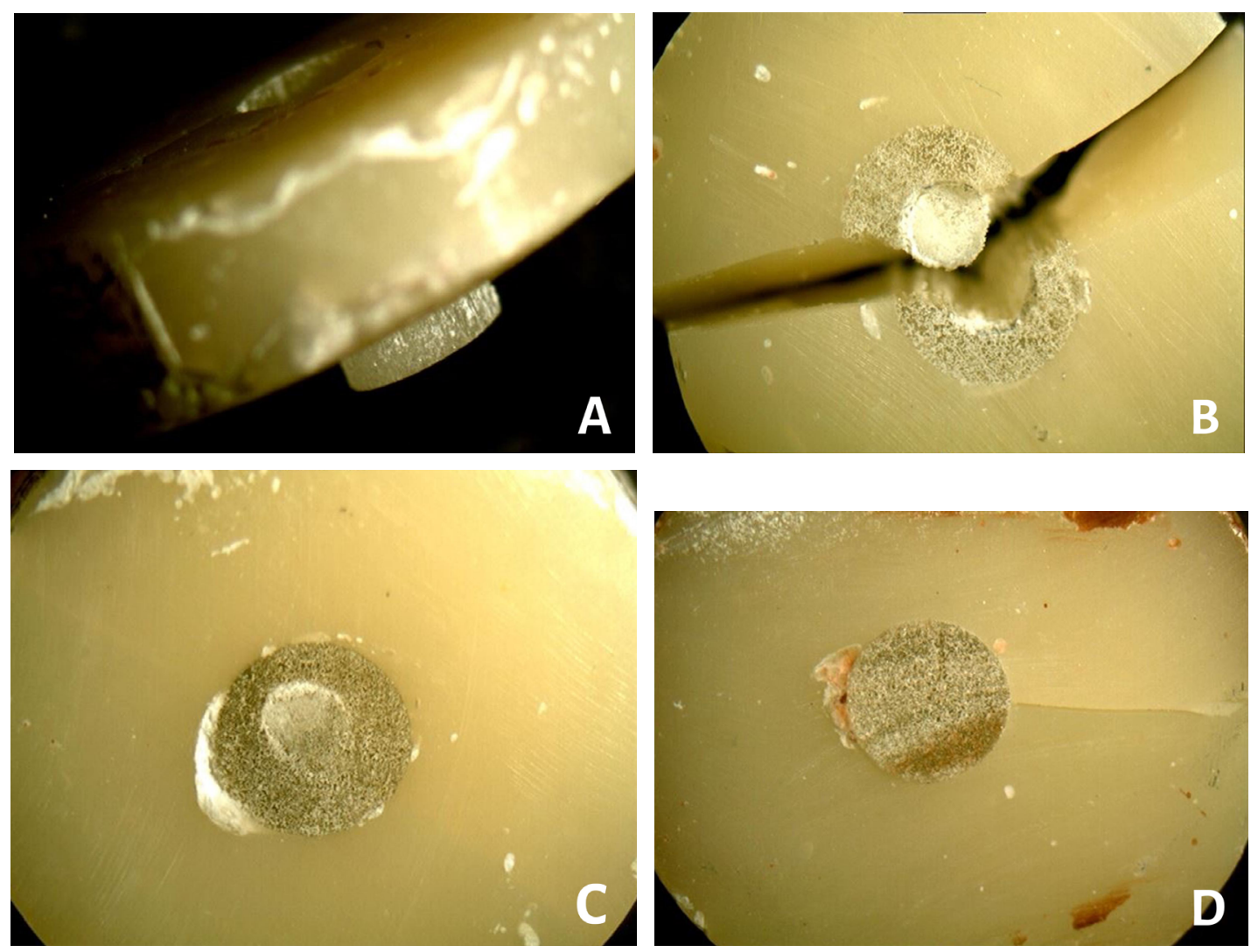

Figura 4: Imagens ilustrativas dos tipos de falha: A) Falha adesiva, B) Falha mista, C) Falha coesiva do pino, D) Falha coesiva da resina.

\section{RESULTADOS}

Na Tabela 1, a análise de variância mostrou indícios de efeitos significativos do tratamento $(P=0,015)$, do terço $(P=0,007)$ e da interação entre fabricante $\times$ tratamento $(P=0,044)$ e tratamento $\times \operatorname{terço~}(P=0,013)$ na resistência de união entre a resina composta e os pinos avaliados. 
Tabela 1: Análise de variância três fatores para resistência de união da resina composta aos pinos avaliados.

\begin{tabular}{lllll}
\hline \multirow{2}{*}{ Efeito } & \multicolumn{2}{l}{ Graus de liberdade } & \multicolumn{2}{l}{ Teste $\mathbf{F}\left(\mathbf{H}_{\mathbf{0}}\right.$ : Médias iguais $)$} \\
\cline { 2 - 5 } & $\mathbf{N u m}$ & $\mathbf{D e n}$ & $\mathbf{F}$ & $\mathbf{P}$ \\
\hline Fabricante & 1 & 31 & 0,55 & 0,464 \\
Tratamento & 2 & 31 & 4,79 & $0,015^{*}$ \\
Terço & 2 & 59 & 5,32 & $0,007^{*}$ \\
Fabricante $\times$ Tratamento & 2 & 31 & 3,47 & $0,044^{*}$ \\
Fabricante $\times$ Terço & 2 & 59 & 1,37 & 0,263 \\
Tratamento $\times$ Terço & 4 & 59 & 3,48 & $0,013^{*}$ \\
Fabricante $\times$ Tratamento $\times$ Terço & 4 & 59 & 2,14 & 0,087 \\
\hline
\end{tabular}

*P $<0.05$ denota diferença estatística significante.

Na Tabela 2, há a comparação das médias de resistência de união da resina composta aos diferentes pinos de fibra de vidro, de acordo com o tratamento de superfície utilizado. Para os pinos Angelus, foi observado que o tratamento com silano (GA-1) apresentou valor significativamente maior (23,10 MPa) que os tratamentos com peróxido (GA-2) (17,19 MPa) e com jateamento (GA-3) (16,28 MPa). Por outro lado, não foram observadas diferenças significativas entre os tratamentos para os pinos FGM $(P>0,05)$.

Tabela 2: Médias (desvio padrão) de resistência de união da resina composta aos diferentes pinos de fibra de vidro, de acordo com os tratamentos de superfície utilizados.

\begin{tabular}{llllllll}
\hline \multirow{2}{*}{ Tratamento } & \multicolumn{7}{c}{ Pino de fibra } \\
\cline { 2 - 8 } & \multicolumn{1}{c}{ Angelus } & FGM \\
\hline 1- Silano & 23,10 & $(5,72)$ & A & 18,45 & $(4,11)$ & A & \\
2 - Peróxido & 17,19 & $(6,94)$ & B & 17,51 & $(6,72)$ & A & \\
3 - Jateamento & 16,28 & $(4,39)$ & B & 18,19 & $(4,25)$ & A \\
\hline
\end{tabular}

Médias com letras iguais na coluna não diferem entre si (teste de Tukey, $\mathrm{P}<0,05$ ).

A Tabela 3 apresenta a comparação das médias de resistência de união nos diferentes terços das amostras avaliadas, de acordo com o tratamento de superfície utilizado. No tratamento com silano, é possível verificar que os terços médio (21,11 MPa) e cervical (22,13 $\mathrm{MPa})$ diferem significativamente do terço apical $(19,08 \mathrm{MPa})(P<0,05)$. No tratamento com peróxido, houve diferença significativa entre o terço médio (21,16 MPa) e apical (12,55 MPa). 
Tabela 3: Médias (desvio padrão) de resistência de união nos diferentes terços das amostras avaliadas, de acordo com o tratamento de superfície utilizado.

\begin{tabular}{lllllll}
\hline Terço & \multicolumn{2}{l}{ 1-Silano } & \multicolumn{2}{l}{ 2 - Peróxido } & \multicolumn{2}{l}{ 3-Jateamento } \\
\hline Apical & 19,08 & $(5,01) \mathrm{B}$ & 12,55 & $(2,26) \mathrm{B}$ & 17,46 & $(5,31) \mathrm{A}$ \\
Médio & 21,11 & $(4,94) \mathrm{A}$ & 21,16 & $(6,82) \mathrm{A}$ & 16,03 & $(4,95) \mathrm{A}$ \\
Cervical & 22,13 & $(6,29) \mathrm{A}$ & 18,34 & $(7,11) \mathrm{AB}$ & 18,22 & $(2,31) \mathrm{A}$ \\
\hline
\end{tabular}

Médias com letras iguais na coluna não diferem entre si (teste de Tukey, $\mathrm{P}<0,05$ ).

Com relação aos tipos de falha, a Tabela 4 apresenta a comparação das proporções de recorrência dos padrões de falha. Foi constatado, 61 casos de falhas mistas, certificando assim, a maior prevalência $(57,01 \%)$. Em seguida, observa-se um total de 38 casos $(35,51 \%)$ de falha coesiva da resina. O número de casos de falha coesiva do pino (7) e adesiva (1) correspondem, respectivamente, a $6,54 \%$ e $0,93 \%$. O teste de qui-quadrado indicou diferença significativa $(P=0,0001)$ entre as proporções de ocorrência dos diversos padrões de falha.

Tabela 4: Frequências, porcentagens de recorrência e teste de qui-quadrado de Pearson para comparar as proporções de recorrência dos padrões de falha.

\begin{tabular}{lllll}
\hline \multirow{2}{*}{ Padrão de Falha } & Frequência & Porcentagem & \multicolumn{2}{c}{ Teste de qui-quadrado } \\
\cline { 5 - 5 } & & & Estatística & $\mathbf{P}$ \\
\hline Adesiva & 1 & 0,93 & & \\
Coesiva da Resina & 38 & 35,51 & & \\
Coesiva do Pino & 7 & 6,54 & & \\
Mista & 61 & 57,01 & & \\
\hline
\end{tabular}

*P< 0.05 denota diferença estatística significante.

A Tabela 5 apresenta a distribuição dos padrões de falha de acordo com os fatores em estudo e, o teste de qui-quadrado permite testar a associação com os fatores do estudo (fabricante, tratamento e terço). $O$ teste de qui-quadrado de razão de verossimilhança não nos dá indícios $(P>0,05)$ da existência de associação entre as distribuições dos padrões de falha e os fabricantes. Observou-se que, em ambos os pinos, a maior parte das falhas foi do tipo mista seguido, em ambos os fabricantes, pelas falhas coesivas da resina e coesivas do pino.

Porém, ainda na Tabela 5, é possível verificar que a análise do teste de qui-quadrado de razão de verossimilhança nos dá indícios $(P<0,05)$ da existência de associação entre o tipo de falha e o tratamento. No tratamento com silano, observou-se ausência de falhas coesivas no pino. No peróxido foram verificadas proporções parecidas entre falha coesiva da resina $(44,44 \%)$ e mista $(47,22 \%)$, diferente do ocorrido nos demais tratamentos, e teve uma proporção intermediária $(8,33 \%)$ de falhas coesivas do pino. No tratamento com jateamento houve uma maior diferença entre as falhas coesivas da resina $(22,86 \%)$ e mistas $(65,71 \%)$ e também maior proporção de falhas coesivas do pino (11,43\%). Por outro lado, ao analisar os padrões de falha e os terços, não houve indícios $(P>0,05)$ da existência de associação entre os mesmos. Observa-se um mesmo padrão de distribuição dos tipos de falha em todos os terços com diferenças que podem ser consideradas como casuais. 
Tabela 5: Frequência (porcentagem) de ocorrência dos diferentes padrões de falha de acordo com as categorias de fabricante, tratamento e terço.

\begin{tabular}{cllll}
\hline \multirow{2}{*}{ Efeito / Nível } & \multicolumn{3}{c}{ Padrão de falha } & $\begin{array}{l}\text { Teste de } \\
\text { qui-quadrado }\end{array}$ \\
\cline { 2 - 4 } & Coesiva da resina & Coesiva do pino & Mista & P \\
\hline Fabricante & & & \\
Angelus & $18(34,62)$ & $2(3,85)$ & $32(61,54)$ & 1,54 \\
FCM & $20(37,04)$ & $5(9,26)$ & $29(53,70)$ & 0,4622 \\
Tratamento & & & & \\
Jateamento & $8(22,86)$ & $4(11,43)$ & $23(65,71)$ & \\
Peróxido & $16(44,44)$ & $3(8,33)$ & $17(47,22)$ & 9,66 \\
Silano & $14(40,00)$ & $0(0,00)$ & $21(60,00)$ & $0,0465^{*}$ \\
Terço & & & & \\
Apical & $13(36,11)$ & $3(8,33)$ & $20(55,56)$ & \\
Médio & $14(41,18)$ & $0(0,00)$ & $20(58,82)$ & 6,15 \\
Cervical & $11(30,56)$ & $4(11,11)$ & $21(58,33)$ & 0,1882 \\
\hline
\end{tabular}

*P< 0.05 denota diferença estatística significante.

\section{DISCUSSÃo}

A hipótese nula do estudo de que os diferentes tratamentos de superfície avaliados não interferem na resistência de união entre resina composta e os pinos de fibra de vidro não foi aceita, visto que foram observadas diferenças entre os grupos. Apesar de não terem sido observadas diferenças entre os tratamentos de superfície para pinos FGM, maior resistência de união ocorreu para pinos Angelus tratados com silano.

Diversas formas de tratamento de superfície têm sido sugeridas com a finalidade de aumentar a adesão entre estrutura dentária, material restaurador e pino de fibra de vidro. Esses tratamentos visam promover uma maior rugosidade de superfície ou maior interação química entre os componentes da interface ${ }^{11-15}$. Em geral, o tratamento de superfície pode ser realizado de três formas: métodos químicos (aplicação de ácidos), métodos físicos (como o jateamento) e associação de métodos físicos e químicos ${ }^{6,11-14}$.

No presente estudo, os valores de resistência de união variaram entre 16,28 e 23,10 MPa (Tabela 2) e foram associados à um baixo percentual de falha coesiva do pino (Tabela 5) e, portanto, a uma maior preservação da estrutura dental e durabilidade da restauração ${ }^{14}$. O tratamento de superfície com silano nos pinos pré fabricados da marca Angelus, apresentou a maior média de resistência de união entre pino de fibra de vidro e resina composta (23,10 MPa), quando comparado ao grupo de peróxido e jateamento. A maior força de união pode ser explicada pelo uso do silano, seguido da aplicação do adesivo para maximizar a união do pino de fibra e resina composta ${ }^{9,15}$. Isso possivelmente ocorreu porque o sistema adesivo, por ser uma resina de baixa viscosidade, foi capaz de penetrar nas porosidades entre as fibras de vidro expostas ao pino ${ }^{1}$, formando um arranjo micromecânico entre o adesivo infiltrado e as fibras expostas, obtendo, além da união química, a retenção micromecânica'. 
Por outro lado, os menores valores de resistência de união observados no tratamento com jateamento (16,28 MPa) para os pinos Angelus, diferem dos resultados reportados por Goracci et al. ${ }^{2}$ e Soares et al. ${ }^{15}$. Os autores afirmaram que o jateamento por óxido de alumínio causa alterações morfológicas capazes de realizar microretenções superficiais que aumentam a adesão à resina composta.

No presente estudo, não foi observado diferença significativa entre os terços médio, cervical e apical no tratamento com jateamento, provavelmente, devido à ocorrência de aumento de irregularidade em toda superfície dos pinos de fibra de maneira equivalente. O jateamento com óxido de alumínio é utilizado a fim de aumentar a rugosidade da superfície pela remoção parcial da matriz epóxica do pino $^{15}$. Embora alguns estudos demonstrem que este tratamento remove apenas a parte epóxica do pino, outros estudos mostram que este tratamento pode levar à danos nas fibras. Radovic et al. ${ }^{16}$ afirmaram que a inclusão de partícula de óxido de alumínio na superfície do pino pelo jateamento diminui as suas propriedades mecânicas, prejudicando o desempenho clínico. Apesar de provavelmente ocorrer aumento na irregularidade de superfície, a presença das partículas impediria o escoamento da resina composta, o que também pode explicar a ausência de diferença significativa entre os terços.

O peróxido de hidrogênio promove remoção parcial da matriz epóxica, quebra as ligações poliméricas, não causa danos as fibras dos pinos e aumenta a resistência de união entre resina composta e pino de fibra ${ }^{1}$. Braga et $a{ }^{17}{ }^{17}$ avaliaram a influência de diferentes tratamentos (peróxido de hidrogênio a $10 \%$ por 10 minutos, peróxido de hidrogênio a $24 \%$ por 1 minuto e jateamento com óxido de alumínio) em comparação ao grupo não tratado na resistência de união de pinos de fibra de vidro à resina composta. Os autores verificaram que grupos tratados apresentaram resistência de união estatisticamente maior que o controle, porém semelhantes entre si. Elsaka ${ }^{18}$ verificou que o tratamento de pinos de fibra de vidro com peróxido de hidrogênio a $10 \%$ por 5 ou 10 minutos resultou em valores de resistência de união estatisticamente semelhantes ao grupo não tratado ou tratado com silano. Porém, maiores valores foram observados quando a concentração de $30 \%$ foi utilizada por 5 ou 10 minutos. Os resultados deste trabalho não suportam tais afirmações, pois o grupo tratado com peróxido de hidrogênio apresentou menor resistência de união (17,19 MPa) que o grupo silano aplicado em pinos da marca Angelus (Tabela 2). Além disso, foram encontrados valores de resistência de união significantemente maiores no terço médio $(21,16 \mathrm{MPa})$ do que no terço apical (12,55 MPa) (Tabela 3). Possivelmente, pode estar associado ao uso de sistema de pino de fibra de vidro com dupla conicidade ${ }^{2}$.

Neste trabalho, também foi constatado que não houve diferença significante das médias entre os tratamentos realizados nos grupos do fabricante da FGM (Tabela 2). Todas as técnicas de tratamento de superfície avaliadas neste estudo tiveram em comum a aplicação do silano. Goracci et al. ${ }^{2}$, Radovic et $a{ }^{16}{ }^{16}$ e Soares et al. ${ }^{15}$ também constataram o efeito positivo na aplicação de silano, como agente promotor de adesão na interface pino e resina composta. Os silanos são compostos orgânicos que contêm silício, podendo ser bifuncionais, ou seja, ter duas extremidades reativas que favorecem a molhabilidade da superfície resultando em formação de pontes químicas entre o substrato orgânico e inorgânicoํ.

É essencial que a força de união entre pino e compósito resinoso seja capaz de resistir às cargas oclusais do paciente em função, visando a longevidade da reabilitação. Porém, embora esse método restaurador apresente boa longevidade clínica, falhas podem ocorrer. No presente estudo, constatou-se maior prevalência de falha do tipo mista, seguida da falha coesiva da resina (Tabela 4), caracterizada por apresentar defeitos como trincas e fraturas no composto resinoso ${ }^{14}$. Clinicamente, este seria o tipo de falha que proporcionaria menor prejuízo ao paciente, pois não afetará a estrutura do remanescente dental e nem mesmo do pino de fibra de vidro. A correção desta falha se dará de maneira mais simples, quando comparada às demais falhas, pois é necessária apenas a reconstrução da restauração em resina ou substituição da mesma. 
O jateamento é capaz de produzir alterações morfológicas nos pinos, causando ranhuras que impediriam o escoamento da resina ${ }^{1}$. Possivelmente, esse fato explicaria a maior incidência de falhas mistas que as falhas coesivas do pino observadas nesse estudo, pois a rugosidade promove uma maior interação entre substrato resinoso e pino de fibra tendo, dessa forma, uma maior possibilidade de promover falhas entre os dois materiais.

A adesão do canal radicular representa o ponto mais crítico da restauração ${ }^{1}$. Assim, o deslocamento do pino no interior do canal radicular é a falha mais comum e acontece pela falha da adesão à dentina do canal radicular, podendo contribuir para a ocorrência de fratura radicular. Os resultados do presente estudo não sustentam essa afirmação, pois houve a ocorrência de apenas uma falha do tipo adesiva (0,93\%) (Tabela 4).

No presente estudo, foi realizada a análise de resistência de união entre dois pinos de fibra de vidro e uma resina composta, porém uma limitação do estudo é a ausência do ensaio de ciclagem mecânica, visando a simulação da resistência da restauração sobre as cargas mastigatória. Novas pesquisas são necessárias para complementar a análise realizada no presente estudo.

\section{CONCLUSÃo}

Baseado nos resultados obtidos neste estudo, conclui-se que o tratamento de superfície com silano nos pinos da marca Angelus resultou em maior resistência de união entre o pino de fibra de vidro e resina composta quando comparados aos demais tratamentos. A falha do tipo mista teve a maior prevalência, seguida da falha do tipo coesiva da resina. Todos os métodos analisados no presente estudo podem ser utilizados como tratamento de superfície nos pinos de fibra de vidro.

\section{CONFLITO DE INTERESSES}

Os autores declaram não haver conflito de interesses.

\section{REFERÊNCIAS}

1. Monticelli F, Grandini S, Goracci C, Ferrari M. Clinical behavior of translucent fiber posts: a 2-year prospective study. Int] Prosthodont. 2003;16(6):593-6.

2. Goracci C, Raffaelli O, Monticelli F, Balleri B, Bertelli E, Ferrari M. The adhesion between prefabricated FRC posts and composite resin cores: microtensile bond strength with and without post-silanization. Dent Mater. 2005;21(5):437-44.

3. Marchionatti AME, Wandscher VF, Rippe MP, Kaizer OB, Valandro LF. Clinical performance and failure modes of pulpless teeth restored with posts: a systematic review. Braz Oral Res. 2017;31:e64.

4. Figueiredo FED, Martins-Filho PRS, Silva ALF. Do metal post-retained restorations result in more root fractures than fiber post-retained restorations? A systematic review and meta-analysis. ] Endod. 2015;41(3):309-16.

5. Novais VR, Simamotos Júnior PC, Rontani RMP, Correr-Sobrinho L, Soares C]. Bond strength between fiber posts and composite resin core: influence of temperature on silane coupling agents. Braz Dent ]. 2012;23(1):8-14.

6. Alshahrani A, Albaqami M, Naji Z, Al-Khunein Y, Alsubaie K, Alqahtani A, et al. Impact of different surface treatment methods on bond strength between fiber post and composite core material. Saudi Dent ]. Forthcoming 2020.

7. Yenise M, Kulunk S. Effects of chemical surface treatments of quartz and glass fiber posts on the retention of a composite resin. J Prosthet Dent. 2008;99(1):38-45.

8. Chen Q, Wei X, Mi YI, Bai Y, Cai Q, Wang XZ. Effect on the bond strengths of glass fiber posts functionalized with polydopamine after etching with hydrogen peroxide. Dent Mater ]. 2015;34(6):740-5. 
9. Braga NMA, Souza-Gabriel AE, Messias DCF, Rached-Junior FJA, Oliveira CF, Silva RB. Flexural properties, morphology and bond strength of fiber-reinforced posts: influence of post pretreatment. Braz Dent ]. 2012;23(6):679-85.

10. Khoroushi M, Mazaher H, Tarighi P, Samimi P, Khalighinejad N. Effect of antioxidants on push-out bond strength of hydrogen peroxide treated glass fiber posts bonded with two types of resin cement. Restor Dent Endod. 2014;39(4):303-9.

11. Perdigão ], Gomes G, Lee IK. The effect of silane on the bond strengths of fiber posts. Dent Mater. 2006;22(8):752-8.

12. Archiana CH, Raju SMK, Yadhav SS, Konagala RK, Manthena SRK, Teja PR. Effect of surface pretreatment and thermal activation of silane coupling agent on bond strength of fiber posts to resin cement. ] Contemp Dent Pract. 2019;20(11): 1293-6.

13. Ferrari M, Vichi A, Grandini S. Efficacy of different adhesive techniques on bonding to root canal walls: An SEM investigation. Dent Mater. 2001;17(5):422-9.

14. Faria MI, Gomes É, João DCM, Filho MS, Filho CBS, Paulino SM. Tensile strength of glass fiber posts submitted to different surface treatments. Braz Dent ]. 2013;24(6):626-9.

15. Soares C], Soares PV, de Freitas Santos-Filho PC, Castro CG, Magalhaes D, Versluis A. The influence of cavity design and glass fiber posts on biomechanical behavior of endodontically treated premolars. ] Endod. 2008;34(8):1015-9.

16. Radovic I, Monticelli F, Goracci C, Cury ÀH, Coniglio I, Vulicevic ZR, et al. The effect of sandblasting on adhesion of a dual-cured resin composite to methacrylic fiber posts: microtensile bond strength and SEM evaluation. ] Dent. 2007;35(6):496-502

17. Braga NMA, Souza-Gabriel AE, Messias DCF, Rached-Junior FJA, Oliveira CF, Silva RG, et al. Flexural properties, morphology and bond strength of fiber-reinforced posts: influence of post pretreatment. Braz Dent ]. 2012;23(6):679-85

18. Elsaka SE. Influence of chemical surface treatments on adhesion of fiber posts to composite resin core materials. Dent Mater. 2013;29(5):550-8. 